\title{
$\mathrm{SDRE}$ 기법을 이용한 비선형 미지입력 관측기 설계와 \\ 반작용 휠의 고장 검출
}

윤형주*, 진재현**

\section{Design of Nonlinear Unknown Input Observer by SDRE Method and Fault Detection of Reaction Wheels}

\author{
Hyungjoo Yoon* and Jaehyun Jin** \\ Korea Aerospace Research Institute*, Sunchon National University**
}

\begin{abstract}
The authors propose a nonlinear unknown input observer to estimate the angular speed of a satellite and to detect faults of reaction wheels. Input values are necessary to estimate the angular speed. Therefore, estimation errors are inevitable if faults occur in actuators or reaction wheels. Unknown input observers are useful to estimate the states of a system without being affected by unknown faults. The authors have designed a nonlinear unknown input observer by using the SDRE method and verified the proposed observer via numerical simulations. In spite of various and simultaneous faults, we have estimated the states and detected faults exactly by the proposed nonlinear unknown input observer.
\end{abstract}

\section{초 록}

위성의 각속도를 추정하고 반작용 휠의 고장을 검출하기 위하여 비선형 미지입력 관측 기를 제안하였다. 위성의 각속도 추정을 위해서 입력 값을 필요로 하는데, 구동기에 고장 이 발생하는 경우에 정확한 각속도 추정이 어렵다. 미지입력 관측기는 미지의 고장에 영 향을 받지 않으면서 상태추정이 가능하다. 본 논문에서는 SDRE 기법을 적용하여 비선형 미지입력 관측기를 설계하였고, 시뮬레이션을 이용하여 제안한 방법을 검증하였다. 다양한 형태로 동시에 고장이 발생하더라도, 각속도 추정 및 고장 검출이 가능하였다.

Key Words : Satellite angular speed estimation(위성 각속도 추정), Nonlinear unknown input observer(비선형 미지입력 관측기), Fault detection(고장 검출), SDRE(state-dependent Riccati equation, 상태변수 연동 Riccati 방정식)

\section{I. 서 론}

위성의 각속도는 자세제어를 위해 필요한 중 요한 변수이다. 일반적으로 자이로 센서를 이용
하여 각속도를 측정하는데, 최근에는 각속도를 추정(estimation)하는 방법을 많이 연구하고 있다 [1-7]. 자이로를 장착하지 않은 경우나, 자이로가 고장 난 경우에 유용한 방안이 된다. 자이로를
† Received: January 19, 2013 Accepted: March 31, 2013

** Corresponding author, E-mail : donworry@scnu.ac.kr http:/ /journal.ksas.or.kr/

pISSN 1225-1348 / eISSN 2287-6871 
장착하지 않으면 비용 절감과 구조의 단순화를 기대할 수 있어, 소형 위성 설계에 적합하다 (SAMPEX 위성, [3]).

각속도 추정을 위하여 사용하는 방법은, 별센 서를 통하여 측정한 자세 값을 직접 미분하여 추 정하거나 새로운 상태변수로 도입하여 각속도와 연동된 운동방정식을 구하고 확장형 Kalman 필 터를 이용하여 각속도를 추정하는 것이다. 추정 성능에 있어서는 두 번째 방법이 더 우수하다[7]. 그러나 이 방법을 적용하기 위해서는 정확한 입 력 값을 알아야 한다. 일반적으로 추정 문제에 있어서 입력은 정확하게 알 수 있다고 가정하지 만, 구동기 고장이 발생하는 경우에는 이 가정이 성립하지 않는다. 구동기 고장 대응의 목적뿐만 아니라 각속도의 정확한 추정을 위해서도 구동기 의 고장을 신속히 진단해야 한다.

반작용 휠의 고장 검출을 위하여 다양한 방안 이 연구되었다. 대부분의 경우 다중모델(multiple model) 이론에 근거하고 있다[8-16]. 다중모델 필 터의 경우 고려하는 모델의 개수가 많을수록 정 확한 추정이 가능하지만, 그만큼 계산이 복잡해 지는 단점이 있다. 예를 들어, 참고문헌 [8]에서 는 반작용 휠이 정지한 경우만 고려하였다. 반작 용 휠이 멈추지 않고 비정상적인 동작을 한다거 나 둘 이상이 고장 난 상황이면, 이를 위해서 모 델을 추가해주어야 하며 반작용 휠의 개수만큼 증가하게 된다. 그만큼 필터의 개수도 증가한다.

본 논문에서는 필터의 개수를 줄이기 위하여 비선형 미지입력 관측기(UIO)를 제안한다. 고장 의 모드에 상관없이 반작용 휠에 발생하는 고장 의 영향을 배제하도록 $\mathrm{UIO}$ 를 설계하고, 추정된 상태변수를 이용하여 고장을 검출한다. 저자는 비선형 시스템에 대한 확장형 UIO를 제안하였다 [17]. 여기에서는 SDRE(state-dependent Riccati equation) 기법을 이용하여 비선형 $\mathrm{UIO}$ 를 설계 한다. 시뮬레이션을 이용하여 제안한 방법을 검 증하였다. 다양한 형태로 그리고 동시에 발생하 는 고장에 대해서도 추정 및 검출이 가능하였다.

\section{II. 운동방정식 및 문제 정의}

\section{1 운동방정식}

\subsection{1 위성 동역학}

벡터 변수는 볼드체로, 스칼라와 행렬 변수는 일반체로 표시하였다. $I$ 와 0 은 단위행렬과 영행 렬이며, 수식에서 크기가 결정된다. $x_{i}$ 는 벡터 $x$ 의 $\mathrm{i}$ 번째 요소이다. 변수 $z$ 의 함수인 행렬 $P(z)$
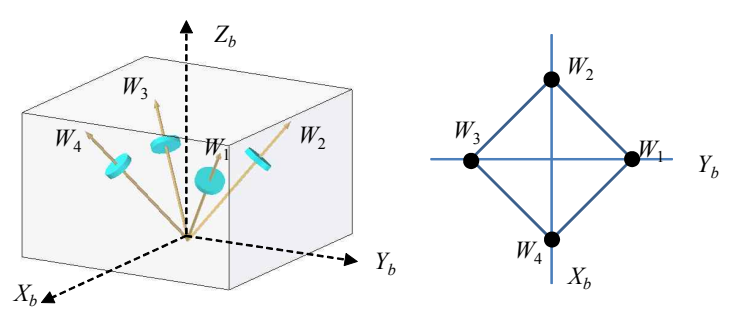

Fig. 1. Configurations of four wheels

와 $Q(z)$ 에 대하여, rank deficiency가 발생하는 $z$ 값이 같으면 $P(z) \sim Q(z)$ 로 표시한다.

대상 위성은 4 개의 반작용 휠을 피라미드 형 상으로 장착한 소형위성이다(Fig. 1 참고). 편의 상, 반작용 휠만 사용한다고 가정한다. 위성의 운 동 방정식은 다음과 같다[18].

$$
\begin{gathered}
\dot{\omega}=J^{-1}\left[J \omega+L J_{W} \Omega\right] \times \omega+J^{-1} L u \\
\dot{\Omega}=-J_{W}^{-1} u=-\frac{1}{J_{w}} u \\
L=\left[\begin{array}{cccc}
0 & -\sin \alpha & 0 & \sin \alpha \\
\sin \alpha & 0 & -\sin \alpha & 0 \\
\cos \alpha & \cos \alpha & \cos \alpha & \cos \alpha
\end{array}\right]
\end{gathered}
$$

$J$ 는 휠을 포함한 위성 전체의 관성모멘트 행 렬이고 대각행렬로 가정한다 $\left(J=\operatorname{diag}\left(J_{1}, J_{2}, J_{3}\right)\right)$. $J_{W}=J_{w} I_{4}$ 는 휠의 관성모멘트 행렬이다. $J_{w}$ 는 휠 의 회전축에 대한 관성모멘트 값이다.

$\omega \in R^{3 \times 1}$ 는 위성의 동체좌표에 대한 각속도 벡터이고, $\Omega \in R^{4 \times 1}$ 는 휠의 각속도 벡터이며, $u \in R^{4 \times 1}$ 는 위성의 제어입력이면서, 휠에 대해서 는 반작용 입력(부호가 반대)이 된다.

반작용 휠의 장착형상에 따라서 입력배분 행 렬 $L$ 이 결정된다. Fig. 1 에서 모든 휠의 회전축 이 $Z_{b}$ 축과 각도 $\alpha$ 만큼 기울어져 있다고 가정하 였다(식 (3) 참고).

\subsection{2 측정방정식}

별센서는, 관성좌표계에 대하여 별센서가 향하 고 있는 방향(방향 벡터)에 대한 정보를 제공한 다. 한 개의 별센서로도 자세를 알 수 있지만, 이 런 경우 특정 축에 대한 정밀도가 저하된다[19]. 두 개의 별센서를 사용하면 이러한 단점을 보완 하여 동일한 정밀도를 보장한다.

측정한 데이터를 신호처리(QUEST나 TRIAD 알고리듬 사용)하여 쿼터니언 값을 제공하는 경 우에는 쿼터니언 변화율 식을 사용한다.

방향 벡터 $\left(b, c \in R^{3 \times 1},\|b\|,\|c\|=1\right)$ 값을 제공하 는 두 개의 센서를 고려하는 경우에, 변화율은 식 (4)와 같이 각속도와 관련된다. 


$$
\dot{b}=b \times \omega=[b \times] \omega, \quad \dot{c}=c \times \omega=[c \times] \omega
$$

$[\times]$ 는 벡터의 외적을 행렬의 곱으로 표현한 것이다. 방향 벡터를 직접 이용하는 것이 신속한 고장 검출에 도움이 된다고 판단되며, 별센서 뿐 만 아니라 지자계 센서 와의 조합에도 적용 가능 하기 때문에[2], 식 (4)를 이용한다.

\subsection{3 문제정의}

별센서의 방향 벡터 측정을 이용하여 각속도 를 추정할 때, 구동기의 고장이 추정성능에 영향 을 미칠 수 있다. 구동기의 고장을 신속히 검출 하여, 고장대응 및 각속도 추정의 성능을 보장해 야 한다.

반작용 휠 고장의 원인은 다양하지만[16], 휠의 속도 이상으로 나타나게 된다. 제어불가한 입력 $(d)$ 이 제어 입력 $\left(u_{c}\right)$ 에 더해진다고 표현한다.

$$
u=u_{c}+d
$$

$d_{i}=-u_{i, c}$ 이면 $\mathrm{i}$ 번째 구동기가 가감속이 이루 어지지 않는다. $d_{i}=-(1-\beta) u_{i, c}$ 이면, 휠의 작용 력이 축소 $(\beta<1)$ 혹은 증가 $(\beta>1)$ 하는 오동작을 뜻한다. $d_{i}=-u_{i, c}+\gamma J_{w} \Omega_{i}$ 로 두면 $(\gamma>0)$, 휠이 점 근적으로 멈추는 것을 의미한다. $\beta$ 와 $\gamma$ 는 적절 한 값을 선택하여 고장을 모사한다. 신뢰성 있는 휠 개발을 위해서는 고장의 원인[16]과 이에 대 한 대응 연구가 필요한 분야이지만, 이에 대한 것은 향후 연구 주제로 다루고자 한다.

식 (1) (5)를 정리하여 다음과 같이 쓴다.

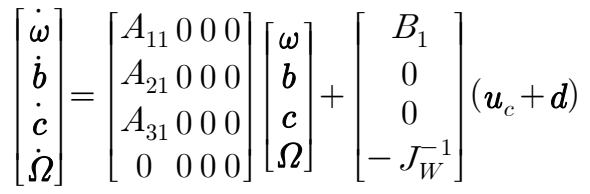

$$
\begin{aligned}
& y=\left[\begin{array}{llll}
0 & I & 0 & 0 \\
0 & 0 & I & 0 \\
0 & 0 & 0 & I
\end{array}\right]\left[\begin{array}{c}
\omega \\
b \\
c \\
\Omega
\end{array}\right] \\
& A_{11}=J^{-1}\left[\left(J \omega+L J_{W} \Omega\right) \times\right], A_{21}=[b \times] \\
& A_{31}=[c \times], B_{1}=J^{-1} L
\end{aligned}
$$

참고문헌 $[8,10-16]$ 에서는 각속도를 측정할 수 있다고 가정하였다. 특히, 참고문헌 $[10,12,15]$ 는 반작용 휠의 내부 모델을 개발하면서, 휠의 각속도와 전류를 상태변수 및 출력으로 선택하였 다. 참고문헌 [9]는 잔차(residual)의 부호 패턴을 보고 고장을 검출하는 방법을 제안하였다. 그러
나 고장이 존재하는 동안에는 올바른 휠 속도와 각속도를 추정할 수 없다. 휠의 각 운동량(또는 각속도)은 $A_{11}$ 에 반영되기 때문에 각속도 추정을 위해서는 반드시 알아야 하는 중요한 변수이다.

\section{2 미지입력 관측기}

\subsection{1 선형 설계이론}

먼저, 선형시스템에 대한 미지입력 관측기 이 론을 소개한다[20-21]. 미지입력 $d$ 가 작용하는 선 형시스템을 고려한다.

$$
\dot{x}=A x+B u+E d, y=C x
$$

상태 추정 $\hat{x}$ 와 관측기를 식 (8)과 같이 설정한 다. $F, L, T, H$ 는 전개과정에서 결정한다.

$$
\begin{gathered}
\hat{x}=z+H y \\
\dot{z}=F z+(L+F H) y+T B u
\end{gathered}
$$

상태 추정 오차를 $e=x-\hat{x}$ 으로 가정할 때, 오 차 동역학은 다음과 같이 전개된다.

$$
\begin{aligned}
\dot{e}=(I-H C)[A x+B u+E d] \\
-F z-(L+F H) y-T B u
\end{aligned}
$$

$T=I-H C, F=T A-L C$ 로 정의하고, 식 (9) 를 정리한다.

$$
\dot{e}=F e+(E-H C E) d
$$

행렬 $H$ 와 $L$ 은 $E-H C E=0, \operatorname{Re}\left(\lambda_{i}(F)\right)<0$ 이 되도록 선정한다. $C E$ 의 우측 역행렬 $\left(E E^{R}=I\right)$ 을 이용하여 $H=E(C E)^{R}$ 로 결정한다. $L$ 은 다음 절 에서 설명한다. 그러면 미지입력 $d$ 에 무관하게 오차가 수렴하여 상태 추정이 이루어진다. 고장 이 발생하더라도 상태추정이 계속 이루어진다는 것이 미지입력 관측기(UIO)의 장점이다. 행렬 $H$ 와 $L$ 이 존재할 조건은 다음과 같다.

C1: $\operatorname{rank}(E)=\operatorname{rank}(C E)$

C2: no unstable transmission zeros of the system $(A, E, C, 0)$

첫째 조건은, 고장 모드가 센서 출력과 직접 연관되는 것을 의미한다. 고장 모드가 검출되는 센서를 decoupling시켜 관측기의 입력에서 배제 한다. 둘째 조건은 $\mathrm{UIO}$ 의 제어불가 극점과 관련 된 것이다. $\mathrm{UIO}$ 와 시스템 역변환 조건은 같다 [21]. 그래서 원 시스템의 영점은 $\mathrm{UIO}$ 의 제어불 가 극점이 된다. $\mathrm{UIO}$ 의 추정값이 수렴하기 위해 서는 불안정한 영점이 없어야 한다. 


\subsection{2 비선형 미지입력 관측기}

추정 대상 시스템은 식 (6)과 같은 비선형 시 스템이다. 비선형 시스템에 대한 추정기법은 확 장형 추정기(확장형 Kalman 필터 등)를 많이 이 용하였다[2,3]. 최근에는 비선형 시스템에 대해 적용할 수 있는 SDRE(state-dependent Riccati equation) 기법에 대한 연구가 수행되고 있다[4, 7, 22-25]. SDRE 기법은 LQ에 대응되는 비선형 제어 기법으로, 체계적으로 비선형 시스템의 제 어와 추정 문제를 다룰 수 있다.

비선형 시스템을 식 (11)의 형태로 정리할 수 있다고 가정한다. 여기서 $A(x), B(x)$ 를 $\mathrm{SDC}$ (state-dependent coefficients)라고 한다.

$$
\dot{x}=A(x) x+B(x) u, y=C x
$$

이에 대한 SDRE 추정기는 식 (12)의 형태가 된다[4, 7, 24].

$$
\begin{gathered}
\dot{\hat{x}}=A(\hat{x}) \hat{x}+B(\hat{x}) u+K(\hat{x})[y-C \hat{x}] \\
K(\hat{x})=P(\hat{x}) C^{T} R^{-1} \\
0=A(\hat{x}) P+P A^{T}(\hat{x})-P C^{T} R^{-1} C P+Q
\end{gathered}
$$

비슷하게, 식 $(10)$ 의 $(T A, C)$ 에 대한 이득행렬 $L$ 을 구한다. 행렬 $Q$ 와 $R$ 은 튜닝를 위해 결정하 는 가중치 행렬이다.

$$
\begin{gathered}
L(\hat{x})=P(\hat{x}) C^{T} R^{-1} \\
0=T A(\hat{x}) P+P A^{T}(\hat{x}) T^{T-} P C^{T} R^{-1} C P+Q
\end{gathered}
$$

비선형 시스템에서 식 (11)의 SDC를 유도하는 과정에서 유일성(uniqueness)을 보장하지 못하기 때문에, 최종적으로 유도된 제어/추정 알고리듬 의 전영역 안정성(global stability)을 보장하지 못 한다[4, 22]. 본 논문의 대상 시스템에 대한 운동 방정식은 이미 SDC 형태로 유도되었다. 그렇지 만 이것이 유일성을 보장하지는 못한다[4]. 이에 대한 연구는 아직까지 진행 중이다.

그리고 매 샘플링 주기마다 식 (13)을 풀어야 한다. 상태변수 값에 따라 SDC가 변하므로 이를 반영하여야 한다. 매번 $\mathrm{ARE}$ 를 풀어야 해서 계산 량이 증가하게 된다. 참고문헌 [22]에서는 이를 위한 빠른 해법을 제시하였다. 미지입력 관측기 설계를 위해서는 매주기 마다 두 조건이 만족되 어야 한다. 이에 대해서는 다음 절에서 검증한다.

\section{III. 구동기 고장 모니터링}

\section{1 단일 고장 검출}

식 (6)을 입력과 고장으로 분리하여 정리한다.

$$
\left[\begin{array}{c}
\dot{\omega} \\
\dot{b} \\
\dot{c} \\
\dot{\Omega}
\end{array}\right]=\left[\begin{array}{cccc}
A_{11} & 0 & 0 & 0 \\
A_{21} & 0 & 0 & 0 \\
A_{31} & 0 & 0 & 0 \\
0 & 0 & 0 & 0
\end{array}\right]\left[\begin{array}{c}
\omega \\
b \\
c \\
\Omega
\end{array}\right]+\left[\begin{array}{c}
B_{1} \\
0 \\
0 \\
-J_{W}^{-1}
\end{array}\right] u_{c}+\left[\begin{array}{c}
B_{1} \\
0 \\
0 \\
-J_{W}^{-1}
\end{array}\right] d
$$

입력과 고장의 행렬이 동일하기 때문에, 식 (8) 에서 $T B=0$ 이 된다. 먼저 $\operatorname{rank}$ 조건(조건 $\mathrm{C} 1$ )은 다음과 같이 만족된다.

$$
C E=\left[\begin{array}{c}
0 \\
0 \\
-J_{W}^{-1}
\end{array}\right] \rightarrow \operatorname{rank}(E)=\operatorname{rank}(C E)=4
$$

영점(transmission zeros)은 다음의 행렬에서 rank가 줄어들게(deficient) 하는 $\mathrm{z}$ 값이다.

$$
\left[\begin{array}{cc}
z I-A-E \\
C & 0
\end{array}\right]
$$

영점은 식 (10)의 행렬 $F$ 의 제어불가한 모드 가 되기 때문에 반드시 안정해야 한다. 즉, $z$ 값 이 있다면 $\operatorname{Re}(z)<0$ 이어야 한다(조건 $\mathrm{C} 2$ ). 행 렬의 기본연산을 이용해서 행렬을 변형하여 $z$ 를 구한다(부록 참고). 결과적으로 식 (16)의 영점은 식 (17) 행렬의 rank를 줄이는 $z$ 값이다.

$$
\left[\begin{array}{c}
z I_{3}-A_{11} \\
A_{21} \\
A_{31}
\end{array}\right]
$$

별센서 두 개가 평행하지 않게 배치되어 있다 면, 행렬 $\left[\begin{array}{l}A_{21} \\ A_{31}\end{array}\right]$ 는 full column rank가 되고, 식 (17)의 rank를 줄이는 $z$ 는 존재하지 않는다.

별센서가 1 개이면(예를 들어 식 (4)의 측정 $b$ 만 존재하는 경우), 식 (17)은 다음과 같다.

$$
\begin{gathered}
{\left[\begin{array}{c}
z I_{3}-A_{11} \\
A_{21}
\end{array}\right]=\left[\begin{array}{c}
\left.z I_{3}-J^{-1}[h \times]\right] \\
{[b \times]}
\end{array}\right] \sim\left[\begin{array}{c}
z J-[h \times] \\
{[b \times]}
\end{array}\right]} \\
h=\left[J \omega+L J_{W} \Omega\right]=\left[h_{1}, h_{2}, h_{3}\right]^{T}
\end{gathered}
$$

부호 ' '는 두 행렬이 같은 $\mathrm{z}$ 값에서 rank deficiency가 발생하는 것을 의미한다. 편의상 방 향 벡터를 $b=[1,0,0]^{T}$ 로 하더라도, 수식의 일반 성은 유지한다. 식 (18)은 다음과 같이 정리된다.

$$
\left[\begin{array}{c}
z J-[h \times] \\
{[b \times]}
\end{array}\right]=\left[\begin{array}{ccc}
J_{1} z & h_{3} & -h_{2} \\
-h_{3} & J_{2} z & h_{1} \\
h_{2} & -h_{1} & J_{3} z \\
0 & 0 & 0 \\
0 & 0 & -1 \\
0 & 1 & 0
\end{array}\right] \sim\left[\begin{array}{c}
z \\
-h_{3} \\
h_{2}
\end{array}\right]
$$


방향 벡터에 수직인 방향으로 움직임이 없다 면 $\left(h_{2}=h_{3}=0\right), z=0$ 의 영점이 생긴다. 우연히, 방향 벡터를 축으로 해서 회전운동하고 있는 경 우이다. 참고문헌 [3]에서 언급한 'unobservable stationary motion'에 해당한다. 그러나 평행하지 않은 두 개의 별센서를 사용하면 이러한 문제점 은 없어지고 영점은 존재하지 않기 때문에 미지 입력 관측기의 설계는 가능하다.

\section{2 시뮬레이션을 통한 검증}

시뮬레이션을 위한 파라미터는 Table 1에 나 타내었다. 입력과 고장은 시간에 따라 변하는 것 으로 가정하였다(식 (20) 참고). 센서의 노이즈는 화이트 가우시안 $\left(\sigma_{b}, \sigma_{c}, \sigma_{\Omega}\right)$ 으로 가정하였다.

$$
\begin{gathered}
u_{c}=\left[\begin{array}{cc}
0.011 \sin (0.11 t) \\
-0.012 \cos (0.03 t) \\
-0.013 \sin (0.05 t) \\
0.014 \cos (0.07 t)
\end{array}\right] \\
\begin{cases}d_{1}=0.02 & 100<t<140 \\
d_{2}=-u_{2} & 120<t<180 \\
d_{3}=-u_{3}+0.1 J_{w} \Omega_{3} & 170<t<200 \\
d_{4}=-0.7 u_{4} & 160<t<220\end{cases}
\end{gathered}
$$

고장은 다음과 같이 추정한다.

$$
\begin{aligned}
d(t-\Delta t) & =E^{L}\left[\dot{\hat{x}}-A(\hat{x}) \hat{x}-B u_{c}\right]_{t-\Delta t} \\
& \simeq E^{L}\left[\frac{\hat{x}^{+}-\hat{x}}{\Delta T}-A(\hat{x}) \hat{x}-B u_{c}\right]_{t-\Delta T}
\end{aligned}
$$

$E^{L}$ 은 $E$ 의 좌측 역행렬 $\left(E^{L} E=I\right)$ 이다. $\hat{x}^{+}$는 $\hat{x}$ 보다 한 스텝 이후의 값이다. 결과적으로 현재 시간보다 한 스텝 이전의 고장 값이 추정된다. 차분하는 과정에서 노이즈의 증폭을 줄이기 위하 여 이동평균을 이용하였다(부록 참고).

Figure 2는 고장 입력, 위성의 쿼터니언, 각속 도, 휠 각속도의 변화를 보인 것이다. Fig. 3과 4 는 행렬 $R$ 의 값을 달리했을 때, 고장 추정 결과 이며, Fig. 5는 두 경우에 대한 각속도 추정오차 의 norm이다. Kalman 필터 이론에 따라, $R$ 을

Table 1. Parameters for simulation

\begin{tabular}{|c|c||c|c|}
\hline Parameters & Unit & Parameters & Unit \\
\hline \hline$J=$ diag[30,30,40] & $\mathrm{kg}-\mathrm{m}^{2}$ & $J_{W}=0.003$ & $\mathrm{~kg}-\mathrm{m}^{2}$ \\
\hline$q_{0}: 0.5,0.2,-0.8,0.27$ & - & $\omega_{0}=[-4,1,2] \times 10^{-2}$ & $\mathrm{rad} / \mathrm{s}$ \\
\hline$\Omega_{0}=[1,2,1,-1] \times 100$ & $\mathrm{rpm}$ & $\alpha=45$ & degree \\
\hline$b_{0}=[1,0,0]$ & - & $c_{0}=[0,1,0]$ & - \\
\hline$Q=I_{13}$ & - & $R=0.1 I_{10} / 100 I_{10}$ & - \\
\hline$\sigma_{b}, \sigma_{c}=0.001$ & & $\sigma_{\Omega}=0.1 / 0.5$ & $\mathrm{rad} / \mathrm{s}$ \\
\hline
\end{tabular}
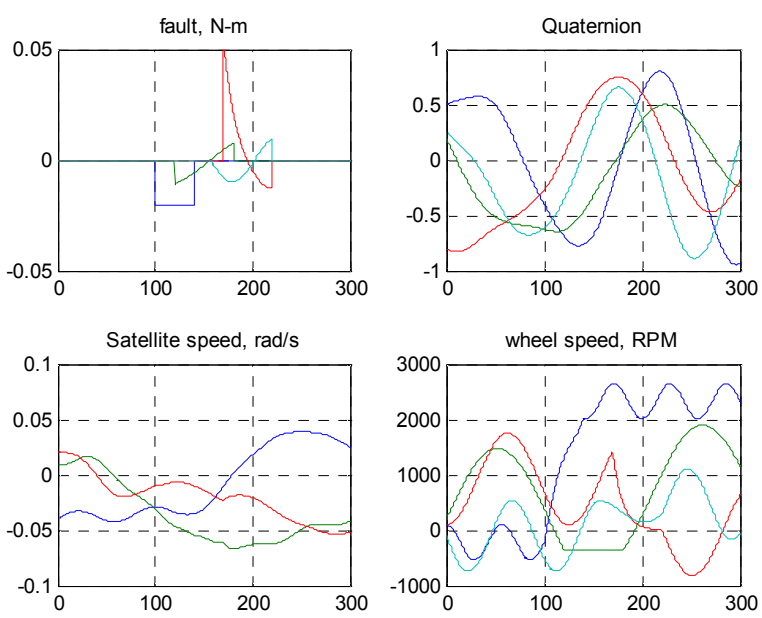

Fig. 2. Reference States of a satellite : faults, quaternion, satellite speeds, wheel speeds.
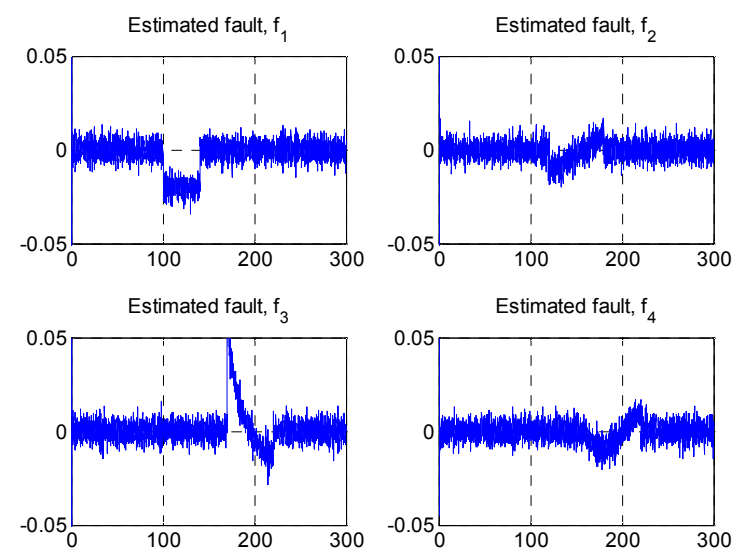

Fig. 3. Simulation results with $R=0.1 I_{3}$, $\sigma_{\Omega}=0.5 \mathrm{rad} / \mathrm{s}:$ satellite speed, faults
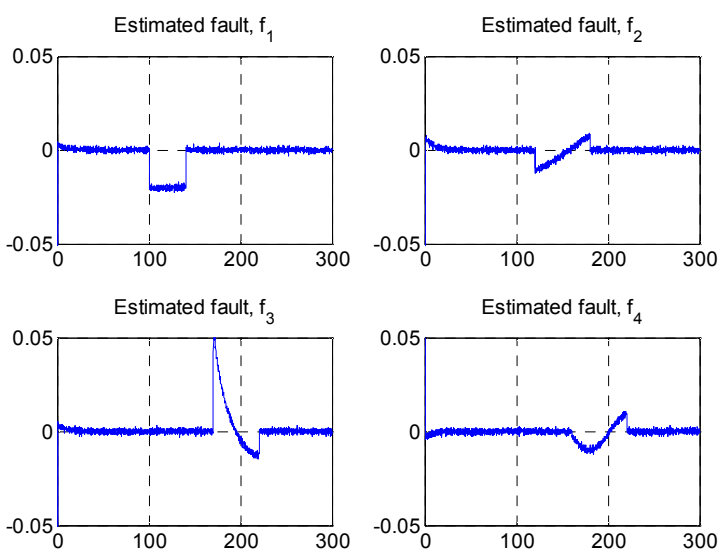

Fig. 4. Simulation results with $R=100 I_{3}$, $\sigma_{\Omega}=0.1 \mathrm{rad} / \mathrm{s}:$ satellite speed, faults 

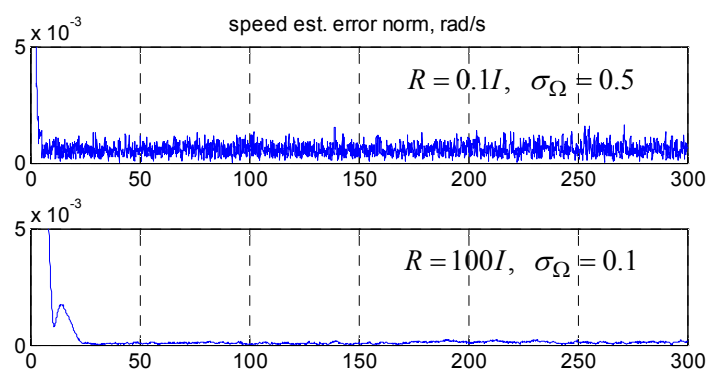

Fig. 5. Simulation results: speed estimation error norms

증가시키면 process 모델에 대한 의존성이 높아 지면서 측정값에 대한 의존도가 줄어든다. 반면 에 $R$ 을 낮추면, 모델 오차 등에 대한 민감도가 줄어든다. 적절한 값을 선정하는 것이 중요하다.

고장에 대한 추정은 휠 각속도 측정 잡음의 영향을 많이 받는다. 고장의 크기가 작은 경우에 는 검출되지 않을 수 있다(fault missing). 그렇지 만 각속도 추정에는 영향을 미치지 않는다. 각속 도 추정은 별센서의 출력과 관련 있기 때문이다 (식 (6) 참고). 고장 검출의 정확도와 고가의 휠 에 대한 투자 간의 적절한 균형이 필요하다.

\section{IV. 결 론}

위성의 각속도를 추정하고 반작용 휠의 고장 을 검출하기 위한 방법으로 비선형 미지입력 관 측기를 제안하였다. 특히, 관측기 설계에 SDRE (State-dependent Riccati Equation) 기법을 적용 하여, 선형시스템에 대한 설계이론을 활용하였다. 다양한 고장을 가정한 시뮬레이션에서 각속도와 고장 추종성능을 검증하였다. 별센서를 가정하였 지만, 지자계 센서와의 조합도 가능하다. 향후, 소형위성에의 응용이 가능할 것으로 기대하며, 이를 위한 추가적인 연구를 진행할 계획이다.

\section{References}

1) Carmi, A., and Oshman, Y., "Fast particle filtering for attitude and angular rate estimation from vector observations," Journal of Guidance, Control, and Dynamics, Vol.32, No.1, 2009, pp.70 78.

2) Tortora, P., et. al. "Spacecraft angular rate estimation from magnetometer data using an analytic predictor," Journal of Guidance, Control, and Dynamics, Vol. 27, No. 3, 2004, pp.365 373.

3) Oshman, Y., and Dellus, F., "Spacecraft angular velocity estimation using sequential observation of a single directional vector," Journal of Spacecraft and Rockets, Vol. 40, No. 2, 2003, pp.237 247.

4) Harman, R., and Bar-Itzhack, I., "Pseudolinear and state-dependent Riccati equation filters for angular rate estimation," Journal of Guidance, Control, and Dynamics, Vol. 22, No. 5, 1999, pp.723 725.

5) Azor, R., et. al., "Satellite angular rate estimation from vector measurements," Journal of Guidance, Control, and Dynamics, Vol. 21, No. 3, 1998, pp. $450 \sim 457$.

6) Bar-Itzhack, I., "Classification of algorithms for angular velocity estimation," Journal of Guidance, Control, and Dynamics, Vol. 24, No. 2, 2001, pp.214 218.

7) Azor, R., et. al., R., "Angular rate estimation using delayed quaternion measurements," Journal of Guidance, Control, and Dynamics, Vol. 24, No. 3, 2001, pp.436 443.

8) Choi, K. and Park, C., "Design of fault isolator of satellite reaction wheel system using dual filter and multi-hypothesis extended Kalman filter," Journal of The Korean Society for Aeronautical and Space Sciences, Vol. 37, No. 12, 2009, pp.1225 1231.

9) Choi, K. and Park, C., "Fault isolation technique of reaction wheels using star tracker measurement," Proceedings of the 2009 KSAS Fall Conference, 2009.11, pp.962 965.

10) Tudoroiu, et. al., "Real-time embedded fault detection estimators in a satellite's reaction wheels," Proceedings of the International Multiconference on Computer Science and Information Technology, 2010, pp.759 766.

11) Tudoroiu, N., and Khorasani, K., "Fault detection and diagnosis for satellite's attitude control system using an interactive multiple model approach," Proceedings of the 2005 IEEE Conference on Control Applications, 2005, pp.1287 1292.

12) Tudoroiu, N., et. al., "Interactive bank of unscented Kalman filters for fault detection and isolation in reaction wheel actuators of satellite attitude control system," Proceedings of the 32nd Annual Conference on IEEE Industrial Electronics, 2006, pp.264 269.

13) Meskin, N., and Khorasani, K., "Fault detection and isolation in a redundant reaction wheels configuration of a satellite," Proceedings of the 
IEEE International Conference on Systems, Man and Cybernetics, 2007, pp.3153 3158.

14) Jiang, T., and Khorasani, K., "A fault detection, isolation and reconstruction strategy for a satellite's attitude control subsystem with redundant reaction wheels," Proceedings of the IEEE International Conference on Systems, Man and Cybernetics, 2007, pp.3146 3152.

15) Talebi, H., et. al., "Fault detection and isolation for uncertain nonlinear systems with application to a satellite reaction wheel actuator," Proceedings of the IEEE International Conference an Systems, Man and Cybernetics, 2007, pp.3140 3145.

16) Tudoroiu, N., and Khorasani, K., "Satellite fault diagnosis using a bank of interacting Kalman filters," IEEE Transactions on Aerospace and Electronic Systems, Vol. 43, No. 4, 2007, pp.1334 1350.

17) Jin, J. and Yong, K., "Fault detection of a spacecraft's reaction wheels by extended unknown input observer," Journal of Institute of Control, Robotics and Systems, Vol. 17, No. 11, 2011, pp.113 8 1144.

18) Bang, H., Tahk, M., and Choi, H., "Large angle attitude control of spacecraft with actuator saturation," Control Engineering Practice, Vol. 11, No. 9, 2003, pp.989 997.

19) Lee, H., "A study on the determination of star sensors mounting direction for remote sensing satellites," Journal of The Korean Society for Aeronautical and Space Sciences, Vol. 35, No. 8, 2007, pp.735 740.

20) Wang, S., et. al., "Observing the states of systems with unmeasurable disturbances," IEEE Transaction on Automatic Control, Vol. 20, No. 5, 1975, pp.716 717.

21) Jin, J, et. al., "Time-delayed state and unknown input observation," International Journal of Control, Vol. 66, 1997, pp. 733-745.
22) Yang, C., et. al., "Research on the Design of Helicopter Nonlinear Optimal Controller using SDRE Technique," Journal of The Korean Society for Aeronautical and Space Sciences, Vol. 36, No. 12, 2008, pp. $1152 \sim 1162$.

23) Kim, S. and Kwon, S., "SDRE Based Nonlinear Optimal Control of a Two-Wheeled Balancing Robot," Journal of Institute of Control, Robotics and Systems, Vol. 17, No. 10, 2011, pp.103 7 1043.

24) Mracek, C., et. al., "A new technique for nonlinear estimation," Proceedings of the 1996 IEEE International Conference on Control Applications, 1996, pp.338 343.

25) Cloutier, J., "State-dependent Riccati equation techniques: an overview," Proceedings of the 1997 American Control Conference, Vol. 2, 1997, pp.932 936.

$$
\text { 부 록 }
$$

- 식 (17) 유도:

$$
\left[\begin{array}{ccccc}
z I_{3}-A_{11} & 0 & 0 & 0 & -B_{1} \\
-A_{21} & z I & 0 & 0 & 0 \\
-A_{31} & 0 & z I & 0 & 0 \\
0 & 0 & 0 & z I & J_{W}^{-1} \\
0 & I & 0 & 0 & 0 \\
0 & 0 & I & 0 & 0 \\
0 & 0 & 0 & I & 0
\end{array}\right] \sim\left[\begin{array}{ccccc}
z I_{3}-A_{11} & 0 & 0 & 0 & 0 \\
A_{21} & 0 & 0 & 0 & 0 \\
A_{31} & 0 & 0 & 0 & 0 \\
0 & 0 & 0 & 0 & J_{W}^{-1} \\
0 & I & 0 & 0 & 0 \\
0 & 0 & I & 0 & 0 \\
0 & 0 & 0 & I & 0
\end{array}\right]
$$

- 차분계산(이동평균 윈도우 사이즈 $=5$ ):

$$
\begin{gathered}
\frac{\hat{x}^{+}-\hat{x}}{\Delta T} \rightarrow \frac{\tilde{x}^{+}-\tilde{x}}{\Delta T}=\frac{\hat{x}(t)-\hat{x}(t-5 \Delta t)}{5 \Delta t} \\
\tilde{x}^{+}=\frac{1}{5}[\hat{x}(t)+\cdots+\hat{x}(t-4 \Delta T)] \\
\tilde{x}=\frac{1}{5}[\hat{x}(t-\Delta t)+\cdots+\hat{x}(t-5 \Delta T)]
\end{gathered}
$$

